\section{Fatores associados à morbidade materna grave em Ribeirão Preto, São Paulo, Brasil: estudo de corte transversal}

\author{
Factors associated with serious maternal \\ morbidity in Ribeirão Preto, São Paulo State, \\ Brazil: a cross-sectional cohort study
}

\section{Factores asociados a la morbilidad materna grave en Ribeirão Preto, São Paulo, Brasil: estudio de corte transversal}

Magna Santos Andrade 1

Livia Pimenta Bonifácio 2

Jazmin Andrea Cifuentes Sanchez 2

Livia Oliveira-Ciabati 2

Fabiani Spessoto Zaratini 2

Ana Carolina Arruda Franzon 2

Vicky Nogueira Pileggi 2

Giordana Campos Braga 2

Mariana Fernandes 2

Carolina Sales Vieira 2

João Paulo Souza 2

Elisabeth Meloni Vieira 2

doi: 10.1590/0102-311X00021821

\title{
Resumo
}

Este estudo investigou os fatores associados à morbidade materna grave entre mulheres atendidas em maternidades públicas do Município de Ribeirão Preto, São Paulo, Brasil. Trata-se de um estudo quantitativo, analitico, transversal. Participaram 1.098 puérperas com parto em uma das quatro maternidades públicas do município. A coleta de dados ocorreu entre 3 de agosto de 2015 e 2 de fevereiro de 2016, a partir de entrevistas face a face, obtenção de informações dos prontuários e dos cartões da gestante. Para a análise de dados, considerou-se como variável dependente a ocorrência de morbidade materna grave, ou seja, quando a mulher era classificada como near miss materno ou condição potencialmente ameaçadora à vida a partir dos critérios de elegibilidade da Organização Mundial de Saúde. Calculou-se a razão do near miss materno, odds ratio (OR), intervalo de 95\% de confiança (IC95\%) e regressão logística múltipla. A razão do near miss materno foi de 3,6 casos por mil nascidos vivos. As complicações ocorreram principalmente na gravidez $(53,8 \%)$ e os distúrbios hipertensivos foram os mais frequentes (49,4\%). A análise de regressão múltipla mostrou associação entre morbidade materna grave e gestação de risco $(O R=4,5$; IC95\%: 2,7-7,7) e com trabalho de parto induzido $(O R=2,1$; IC95\%: 1,2-3,9). A ocorrência de morbidade materna grave, principalmente na gestação, com destaque para as síndromes hipertensivas, aponta para a necessidade de melhor rastreamento e manejo da elevação dos níveis pressóricos no pré-natal. A associação entre morbidade materna grave e gestação de risco também remonta ao pré-natal, para a demanda de uma maior atenção às mulheres classificadas como risco gestacional. A qualidade da assistência é ponto chave para o enfretamento da morbimortalidade materna no país.

Near Miss; Morte Materna; Morbidade; Saúde Materna

\author{
Correspondência \\ M. S. Andrade \\ Universidade do Estado da Bahia. \\ Rod. Lomanto Júnior, BR 407, Km 127, s/n, Senhor do Bonfim, \\ BA 48970-000, Brasil. \\ magnaenf@yahoo.com.br \\ 1 Universidade do Estado da Bahia, Senhor do Bonfim, Brasil. \\ 2 Universidade de São Paulo, Ribeirão Preto, Brasil.
}




\section{Introdução}

Estima-se se que, em 2017, aproximadamente 295 mil mulheres morreram em todo o mundo em decorrência de complicações no ciclo gravídico-puerperal. Nesse mesmo ano, a razão de mortalidade materna (RMM) em regiões de alta renda, como a Europa, foi de 10 óbitos maternos por 100 mil nascidos vivos, enquanto áreas carentes como a África Subsaariana apresentaram RMM de 547 mortes por 100 mil nascido vivos 1 .

A mortalidade materna reflete as desigualdades entre países ricos e pobres, além de refletir as iniquidades no interior dos próprios países, e o risco de morrer durante a gestação, parto ou pós-parto aumenta proporcionalmente à diminuição das condições socioeconômicas de uma região. Melhores condições de vida e acesso a serviços de saúde de qualidade poderiam evitar aproximadamente $98 \%$ desses óbitos, por isso, muitas mortes maternas são resultado de graves violações dos direitos humanos 2 .

Em 2019, estimativas do Ministério da Saúde brasileiro mostraram que a RMM no Brasil foi de 55,3 mortes por 100 mil nascidos vivos. A meta de redução da mortalidade materna no país para o ano de 2015, segundo os Objetivos de Desenvolvimento do Milênio, era de 35 óbitos por 100 mil nascidos vivos. Entretanto, apesar de uma importante redução do indicador nacional desde 1990, quando ocorriam 140 óbitos maternos por 100 mil nascidos vivos, as estratégias implementadas até o momento não foram suficientes para o alcance da meta proposta para o país 3,4.

Para o enfrentamento da mortalidade materna e a melhoria da saúde destas mulheres, é necessário conhecer e intervir sobre os determinantes de saúde que atuam durante o período gravídico-puerperal. Mas apenas a pesquisa dos óbitos nesse grupo de mulheres é frequentemente insuficiente, devido ao reduzido número absoluto e atipias das mortes maternas, particularmente em países de renda média e alta 5,6 .

Por isso, recomenda-se o estudo das condições potencialmente ameaçadoras à vida que consistem em graves complicações maternas durante a gestação, parto e pós-parto e nos casos de mulheres que quase morreram nesse mesmo período, mas sobreviveram, o near miss materno. A pesquisa dos casos de condições potencialmente ameaçadoras à vida e near miss materno permite um maior volume de informações e produz valioso conhecimento sobre a qualidade da assistência obstétrica e os fatores que podem levar à morte 7 .

A Organização Mundial da Saúde (OMS) padronizou critérios para identificação dos casos de near miss materno e indicadores de qualidade da assistência baseados nas complicações maternas graves. Tal iniciativa objetivou estruturar um sistema internacional de classificação do near miss materno e viabilizar a comparação da saúde materna entre diferentes regiões e países. Acredita-se que esta abordagem possa contribuir para a identificação de problemas prioritários na assistência obstétrica e, com isso, possibilitar o aperfeiçoamento do cuidado 8.

Pesquisa realizada na América Latina, África, Ásia e Oriente Médio identificou 8,3 casos de near miss materno por mil nascidos vivos 9 . No Brasil, dois grandes estudos multicêntricos apresentaram 9,4 e 10,2 casos de near miss materno por mil nascidos vivos 10,11. A pesquisa Nascer no Brasil mostrou associação entre near miss materno e ausência de cuidado pré-natal, busca por dois ou mais serviços de saúde para o parto, complicações obstétricas e cesárea eletiva 11.

Dados confiáveis sobre as complicações maternas graves podem contribuir para a tomada de decisão desde o nível macro até as localidades menores. Este conhecimento viabiliza a adequada alocação de recursos, o planejamento e a implementação de intervenções para a redução dos indicadores de morbimortalidade 12 .

Este estudo teve como objetivo investigar os fatores associados à morbidade materna grave entre mulheres atendidas em maternidades públicas do Município de Ribeirão Preto, em São Paulo, Brasil. 


\section{Métodos}

\section{Desenho do estudo}

Estudo quantitativo, analítico, do tipo transversal.

\section{População, local e período de estudo}

Este estudo integrou uma pesquisa maior, denominada Pré-Natal no Celular (PRENACEL), um ensaio clínico aleatorizado por conglomerados, realizado com gestantes para verificar o impacto de um sistema de distribuição de conteúdo por mensagens via celular (short message service - SMS) para a adesão das mulheres às práticas recomendadas na assistência pré-natal pelo Ministério da Saúde (realização de consultas, exames, vacinas, alimentação saudável, identificação de sinais e sintomas de complicações etc.) 13 .

O PRENACEL foi realizado com gestantes residentes nas áreas de abrangência de 20 unidades básicas de saúde (UBS) de Ribeirão Preto, sendo 10 UBS alocadas como unidades de intervenção (envio de SMS durante a gestação) e 10 UBS como unidades controle (realização do pré-natal padrão) 13.

Em 2020, a população estimada do Município de Ribeirão Preto era de 711.825 habitantes, com um elevado Índice de Desenvolvimento Humano (IDH) de 0,8 14, demonstrando uma melhor situação de educação e renda se comparado à maior parte dos municípios brasileiros.

Para a verificação dos impactos do PRENACEL, todas as mulheres que realizaram o pré-natal nas 20 UBS alocadas para o estudo (unidades controle e intervenção) e que tiveram o parto em uma das quatro maternidades públicas da cidade foram entrevistadas após o parto (Centro de Referência da Saúde da Mulher - Mater, Hospital das Clínicas da Faculdade de Medicina de Ribeirão Preto - Universidade de São Paulo, Santa Casa de Misericórdia de Ribeirão Preto, e Maternidade Cidinha Bonini - Universidade de Ribeirão Preto) 13.

A coleta de dados ocorreu entre 3 de agosto de 2015 e 2 de fevereiro de 2016.

A partir das mulheres entrevistadas nas maternidades para o estudo PRENACEL, foi realizada a análise transversal. Nesta análise, adotou-se o termo morbidade materna grave para representar o conjunto dos casos de condições potencialmente ameaçadoras à vida e near miss materno.

Os critérios de inclusão para a análise foram: internação em uma das quatro maternidades públicas do município; ter realizado ao menos uma consulta pré-natal em 1 das 20 UBS integrantes do estudo PRENACEL; estar no período de pós-parto; idade $\geq 18$ anos; ter desenvolvido morbidade materna grave (condições potencialmente ameaçadoras à vida ou near miss materno) na gestação, parto ou após o parto, segundo dados do prontuário. Para a identificação dos casos de condições potencialmente ameaçadoras à vida ou near miss materno, foram adotados os critérios estabelecidos pela OMS 7 .

Foram excluídas as situações de abortamento e gestação ectópica, pois não entraram no estudo PRENACEL. Também não entraram nesta análise as mulheres que receberam a intervenção PRENACEL (mensagens de texto via celular) devido à potencial interferência da intervenção na redução de morbidade materna grave.

\section{Variáveis, fontes de dados e mensuração}

Por um período de seis meses, a equipe de pesquisa realizou visitas diárias às quatro maternidades. Os prontuários das mulheres internadas no pós-parto eram revisados e os profissionais de saúde envolvidos no cuidado também eram abordados para a busca de mulheres elegíveis. Nos prontuários, procurava-se a presença de qualquer um dos critérios estabelecidos pela OMS para a classificação em condições potencialmente ameaçadoras à vida ou near miss materno, com o objetivo de identificar os casos de morbidade materna grave ${ }^{7}$.

Para evitar perdas dos casos de morbidade materna grave, foi implementado um formulário com todos os critérios de classificação preconizados pela OMS para condições potencialmente ameaçadoras à vida e nera miss materno ${ }^{7}$. Simultaneamente, na caderneta da gestante (documento da mulher com dados do pré-natal), era identificada a UBS onde fez o pré-natal, para verificar se fazia parte do estudo PRENACEL. 
As variáveis utilizadas neste estudo foram obtidas do instrumento de coleta de dados do PRENACEL, preenchido a partir de dados do prontuário, caderneta da gestante e entrevista face a face 13 .

A variável dependente deste recorte transversal foi a ocorrência de morbidade materna grave.

As variáveis independentes utilizadas foram: sociodemográficas (idade, escolaridade, situação marital, raça/cor, exerce trabalho remunerado, chefe de família e classe social definida pelo Critério Brasil 15); antecedentes obstétricos (gestações e partos anteriores, cesarianas e partos vaginais prévios, abortos prévios, natimortos anteriores, último intervalo interpartal e complicações em gestações anteriores); assistência pré-natal e parto (planejamento da gestação, realização do pré-natal, número de consultas pré-natal, pré-natal adequado, idade gestacional na primeira consulta, local do pré-natal (onde realizou a maior parte das consultas pré-natal), gestante de risco, risco atual devido a hipertensão, diabetes, sangramento e infecção urinária, tipo de gestação, tipo de parto e presença de trabalho de parto).

A variável "pré-natal adequado" foi elaborada com base nas recomendações do Ministério da Saúde brasileiro. Considerou-se o mínimo de consultas estabelecidas para cada período gestacional (mínimo de seis consultas, sendo a primeira realizada até o final do primeiro trimestre, duas no segundo e três no terceiro trimestre) 16 .

O instrumento de coleta de dados do PRENACEL foi testado por meio de um estudo piloto conduzido antes do início da coleta de dados (julho de 2015). A equipe de coleta de dados foi formada por oito entrevistadoras que receberam treinamento prévio e foram divididas em quatro grupos para atuação em cada unidade hospitalar.

Em cada instituição, uma supervisora era responsável pelo campo de coleta, realizando a verificação dos instrumentos para avaliar possíveis erros de preenchimento. Em casos de erros de informação, o questionário retornava para a entrevistadora, que voltava a campo para correção das inconsistências.

\section{Tamanho amostral}

O PRENACEL consistiu em um grande estudo, com diversos recortes de pesquisa, a exemplo do ensaio clínico referido e deste estudo transversal. Todas as puérperas que fizeram o pré-natal em 1 das 20 UBS alocadas para o PRENACEL foram entrevistadas nas quatro maternidades para verificar os efeitos da intervenção, totalizando 1.214 participantes.

Para este recorte sobre complicações maternas graves, foram utilizadas as mulheres entrevistadas nas quatro maternidades, sendo excluídas as 116 que receberam SMS referentes à intervenção PRENACEL.

Tal exclusão ocorreu pois apenas três $(2,6 \%)$ mulheres das 116 do grupo intervenção tiveram morbidade materna grave, enquanto 91 (8,3\%) das 10.098 integrantes do grupo não intervenção tiveram morbidade materna grave.

Com isso, há uma diferença significante entre as proporções de adoecimento grave ao comparar os dois grupos ( $\mathrm{p}=0,028)$ e uma razão de chances igual a 3,2, ou seja, a chance de uma mulher que não recebeu mensagens de texto do estudo PRENACEL ter morbidade materna grave é 3,2 vezes maior se comparada à chance de uma mulher que recebe as mensagens.

Nenhuma diferença significante foi observada entre as variáveis independentes ao comparar os dois grupos, o que reforça a hipótese de que a intervenção pode ter tido um efeito protetor no grupo de mulheres que recebeu mensagem de texto, por estimular uma maior adesão das gestantes ao prénatal e ao autocuidado.

Com isso, 1.098 mulheres participaram desta análise.

\section{Análise estatística}

A digitação dos dados ocorreu no Research Eletronic Data Capture (REDCap; https://www.projectredcap.org/) 17. Em seguida, o banco de dados foi transportado para o software Stata, versão 9.1 (https://www.stata.com), processado e analisado. As inconsistências no banco de dados foram investigadas por meio de um conjunto de regras de validação/verificação cruzada. 
Os erros observados foram corrigidos através da verificação do formulário de papel e conferência do prontuário hospitalar. Em situações específicas, em que os problemas não puderam ser resolvidos, as variáveis inconsistentes foram anuladas e consideradas como informação faltante.

A análise descritiva foi realizada a partir das frequências simples e relativas das variáveis pesquisadas

Para examinar as associações entre as variáveis independentes e a morbidade materna grave (variável desfecho) foram calculados odds ratio (OR), intervalo de 95\% de confiança (IC95\%) e testes qui-quadrado ou teste de Fisher.

Quando o valor de p dessas associações, no teste qui-quadrado/Fisher, foi inferior a 0,25, a variável foi incluída no modelo de regressão logística múltipla não condicional - idade, cor da pele, trabalho remunerado, total de parto anteriores, local do pré-natal, ser gestante de risco, tipo de gestação (única ou múltipla), tipo de parto (vaginal ou cesariana) e presença de trabalho de parto.

As variáveis número de gestações anteriores e partos anteriores referem-se a informações semelhantes e optou-se pela variável total de partos anteriores para a análise de regressão, para evitar colinearidade. Só entraram na análise múltipla as mulheres que possuíam todas as variáveis com dados válidos no banco de dados. O nível de significância considerado para a regressão foi de 5\%.

\section{Aspectos éticos}

Este estudo foi aprovado pelo Comitê de Ética em Pesquisa do Centro de Saúde Escola da Faculdade de Medicina de Ribeirão Preto, Universidade de São Paulo (protocolo no 971.458).

Antes da realização das entrevistas, todas as participantes eram orientadas sobre os objetivos do estudo e, em seguida, assinavam o Termo de Consentimento Livre e Esclarecido.

\section{Resultados}

Dentre as 1.098 mulheres que participaram do estudo, 8,3\% desenvolveram morbidade materna grave durante o ciclo gravídico puerperal (quatro casos de near miss materno e 87 de condições potencialmente ameaçadoras à vida), 53,8\% apresentaram o primeiro critério de morbidade materna grave durante a gestação, $26,4 \%$ no parto e $19,8 \%$ até 42 dias de pós-parto.

A razão do near miss materno foi de 3,2 casos por mil nascidos vivos. Não ocorreram casos de óbitos maternos. Os critérios diagnósticos mais frequentes entre as mulheres que desenvolveram morbidade materna grave foram os distúrbios hipertensivos (Tabela 1).

\section{Tabela 1}

Condições diagnosticadas para condições potencialmente ameaçadoras à vida e near miss materno entre mulheres participantes da análise de corte transversal. Município de Ribeirão Preto, São Paulo, Brasil, 2015-2016.

\begin{tabular}{lcccc}
\hline Grupos de critérios & $\begin{array}{c}\text { Condições potencialmente ameaçadoras à saúde }(\mathbf{n}=\mathbf{8 7}) \text { * } \\
\text { \% }\end{array}$ & $\mathbf{n}$ & $\begin{array}{c}\text { Near miss materno }(\mathbf{n}=\mathbf{4}) \text { * } \\
\mathbf{n}\end{array}$ \\
\hline Doenças hemorrágicas & 19 & 21,8 & - \\
Síndromes hipertensivas & 43 & 49,4 & - \\
Indicadores de manejo & 37 & 42,5 & - & - \\
Outras doenças sistêmicas & 22 & 25,3 & 1 & - \\
Critérios Clínicos & - & - & 2 & - \\
Critérios laboratoriais & - & - & 4 & 50,0 \\
Critérios de Manejo & - & - & 100,0 \\
\hline
\end{tabular}

* A soma totalizou mais de 100\%, pois algumas mulheres eram elegíveis para mais de um grupo de critérios de elegibilidade para condições potencialmente ameaçadoras à vida ou near miss materno. Os grupos de critérios apresentados na tabela seguiram a recomendação da Organização Mundial da Saúde 1. 
Em relação ao perfil sociodemográfico das entrevistadas, 87,4\% possuíam menos de 35 anos de idade, 80,8\% tinham 8 anos ou mais de escolaridade, 79,9\% viviam com companheiro, 59,7\% se autodeclararam pretas ou pardas, 53,4\% realizavam trabalho remunerado e 58,5\% pertenciam à classe média (classe C) (Tabela 2).

No que se refere aos antecedentes obstétricos, 47,7\% possuíam 1 ou 2 partos anteriores, dentre estes predominaram os partos vaginais (67,6\%), 73,8\% negaram episódio de abortos prévios e 97,6\% histórico de natimortos anteriores, $92 \%$ referiram último intervalo interpartal maior ou igual a dois anos e 79,6\% negaram complicações em gestações anteriores (Tabela 3).

Sobre a assistência ao pré-natal e ao parto, 58,5\% não panejaram a gestação atual, todas fizeram acompanhamento pré-natal, e $85,2 \%$ fizeram seis ou mais consultas, $68,3 \%$ iniciaram o pré-natal no primeiro trimestre, 74,7\% referiram que a gestação não foi classificada como risco e $67,6 \%$ tiveram o parto vaginal como o tipo de parto atual (Tabela 4).

\section{Tabela 2}

Distribuição das características sociodemográficas de mulheres participantes da análise de corte transversal. Município de Ribeirão Preto, São Paulo, Brasil, 2015-2016.

\begin{tabular}{|c|c|c|c|c|c|}
\hline $\begin{array}{l}\text { Características } \\
\text { sociodemográficas }\end{array}$ & $\begin{array}{l}\text { Total } \\
\text { n (\%) }\end{array}$ & $\begin{array}{c}\text { Mulheres com } \\
\text { morbidade } \\
\text { materna grave * } \\
\text { n (\%) }\end{array}$ & $\begin{array}{l}\text { Mulheres sem } \\
\text { morbidade } \\
\text { materna grave } \\
n(\%)\end{array}$ & OR (IC95\%) & Valor de $p$ ** \\
\hline Idade (anos) & & & & & 0,042 \\
\hline$<35$ & $936(87,4)$ & $70(80,5)$ & $866(88,0)$ & 1,0 & \\
\hline$\geq 35$ & $135(12,6)$ & $17(19,5)$ & $118(12,0)$ & $1,8(1,0-3,1)$ & \\
\hline Escolaridade (anos) & & & & & 0,481 \\
\hline$<8$ & $203(19,2)$ & $19(22,1)$ & $184(19,0)$ & $1,2(0,7-2,1)$ & \\
\hline$\geq 8$ & $853(80,8)$ & $67(77,9)$ & $786(81,0)$ & 1,0 & \\
\hline Situação marital & & & & & 0,355 \\
\hline Com companheiro & $854(79,9)$ & $72(83,7)$ & $782(79,5)$ & 1,0 & \\
\hline Sem companheiro & $215(20,1)$ & $14(16,3)$ & $201(20,5)$ & $0,8(0,4-1,4)$ & \\
\hline Raça/Cor & & & & & 0,064 \\
\hline Preta/Parda & $637(59,7)$ & $60(69,0)$ & $577(58,8)$ & $1,6(1,0-2,5)$ & \\
\hline Outras *** & $431(40,3)$ & $27(31,0)$ & $404(41,2)$ & 1,0 & \\
\hline Trabalho remunerado & & & & & 0,232 \\
\hline Sim & $546(53,4)$ & $39(47,0)$ & $507(53,8)$ & $0,8(0,5-1,2)$ & \\
\hline Não & $479(46,7)$ & $44(53,0)$ & $435(46,2)$ & 1,0 & \\
\hline Chefe de família & & & & & 0,685 \\
\hline A própria mulher & $225(22,9)$ & $20(24,4)$ & $205(22,8)$ & $1,0(0,6-1,7)$ & \\
\hline Companheiro & $539(54,8)$ & $47(57,3)$ & $492(54,6)$ & 1,0 & \\
\hline Outra pessoa & $219(22,3)$ & $15(18,3)$ & $204(22,6)$ & $1,3(0,7-2,4)$ & \\
\hline Classe social \# & & & & & 0,898 \\
\hline$A$ ou $B$ & $111(12,7)$ & $10(13,3)$ & $101(12,6)$ & 1,0 & \\
\hline C & $512(58,5)$ & $42(56,0)$ & $470(58,8)$ & $0,9(0,4-1,9)$ & \\
\hline D ou $E$ & $252(28,8)$ & $23(30,7)$ & $229(28,6)$ & $1,0(0,5-2,2)$ & \\
\hline
\end{tabular}

IC95\%: intervalo de 95\% de confiança; OR: odds ratio.

* Condições potencialmente ameaçadoras à vida/near miss materno;

** Teste qui-quadrado ou Fisher;

*** Branca, indígena e oriental/amarela.

\# Renda mensal média das classes A (> 20 salários-mínimos), B (> 10 e $\leq 20$ salários-mínimos), C ( 4 e $\leq 10$ salários-mínimos), D (> 2 e $\leq 4$ salários-mínimos) e E ( 2 salários-mínimos). O salário do Brasil na época da pesquisa era de BRL 880,00 (USD 267,81). 
Tabela 3

Distribuição dos antecedentes obstétricos de mulheres participantes da análise de corte transversal. Município de Ribeirão Preto, São Paulo, Brasil, 2015-2016.

\begin{tabular}{|c|c|c|c|c|c|}
\hline Antecedentes obstétricos & $\begin{array}{l}\text { Total } \\
\text { n (\%) }\end{array}$ & $\begin{array}{c}\text { Mulheres com morbidade } \\
\text { materna grave * } \\
\text { n (\%) }\end{array}$ & $\begin{array}{c}\text { Mulheres sem morbidade } \\
\text { materna grave } \\
\mathrm{n}(\%)\end{array}$ & OR (IC95\%) & Valor de $p * *$ \\
\hline Gestações anteriores & & & & & 0,126 \\
\hline 0 & $352(34,1)$ & $27(31,4)$ & $325(33,5)$ & 1,0 & \\
\hline $1-2$ & $471(45,7)$ & $35(40,7)$ & $463(47,6)$ & $0,9(0,5-1,5)$ & \\
\hline$\geq 3$ & $208(20,2)$ & $24(27,9)$ & $184(18,9)$ & $1,6(0,9-2,8)$ & \\
\hline Partos anteriores & & & & & 0,064 \\
\hline 0 & $414(39,1)$ & $34(39,5)$ & $380(39,1)$ & 1,0 & \\
\hline $1-2$ & $504(47,7)$ & $34(39,5)$ & $470(48,4)$ & $0,8(0,5-1,3)$ & \\
\hline$\geq 3$ & $140(13,2)$ & $18(20,9)$ & $122(12,6)$ & $1,7(0,9-3,0)$ & \\
\hline Cesarianas prévias & & & & & 0,174 \\
\hline Sim & $231(32,7)$ & $24(40,7)$ & $207(32,0)$ & $1,5(0,9-2,5)$ & \\
\hline Não & $475(67,3)$ & $35(59,3)$ & $440(68,0)$ & 1,0 & \\
\hline Partos vaginais prévios & & & & & 0,406 \\
\hline Sim & $477(67,6)$ & $37(62,7)$ & $440(68,0)$ & $0,8(0,5-1,4)$ & \\
\hline Não & $229(32,4)$ & $22(37,3)$ & $207(32,0)$ & 1,0 & \\
\hline Abortos prévios & & & & & 0,652 \\
\hline Sim & $185(26,2)$ & $14(23,7)$ & $171(26,4)$ & $0,9(0,5-1,6)$ & \\
\hline Não & $521(73,8)$ & $45(76,3)$ & $476(73,6)$ & 1,0 & \\
\hline Natimortos anteriores & & & & & 0,161 \\
\hline Sim & $17(2,4)$ & $3(5,1)$ & $14(2,2)$ & $2,4(0,7-8,7)$ & \\
\hline Não & $689(97,6)$ & $56(94,9)$ & $633(97,8)$ & 1,0 & \\
\hline Intervalo interpartal (anos) & & & & & 0,548 \\
\hline$<2$ & $47(8,0)$ & $5(10,2)$ & $42(7,8)$ & $1,4(0,5-3,6)$ & \\
\hline$\geq 2$ & $542(92,0)$ & $44(89,8)$ & $498(92,2)$ & 1,0 & \\
\hline Complicações em gestações anteriores & & & & & $<0,001$ \\
\hline Sim & $129(20,4)$ & $24(43,6)$ & $105(18,2)$ & $3,5(2,0-6,2)$ & \\
\hline Não & $502(79,6)$ & $31(56,4)$ & $471(81,8)$ & 1,0 & \\
\hline
\end{tabular}

IC95\%: intervalo de 95\% de confiança; OR: odds ratio.

* Condições potencialmente ameaçadoras à vida/near miss materno;

** Teste qui-quadrado ou Fisher.

$\mathrm{Na}$ análise de associação pelo qui-quadrado, a morbidade materna grave associou-se à idade materna $\geq 35$ anos $(\mathrm{p}=0,042)$ (Tabela 1$)$, ter apresentado complicações em gestações anteriores $(\mathrm{p}<0,001)$ (Tabela 2), gestação de risco na gravidez atual $(\mathrm{p}<0,001)$, risco atual devido à hipertensão $(\mathrm{p}<0,001)$, gestação atual gemelar $(\mathrm{p}=0,003)$, cesárea atual $(\mathrm{p}<0,001)$ e trabalho de parto induzido $(\mathrm{p}<0,001)$ (Tabela 4).

Na análise múltipla, observou-se associação entre morbidade materna grave com o risco na gestação atual (OR = 4,5; IC95\%: 2,7-7,7) e trabalho de parto induzido (OR = 2,1; IC95\%: 1,2-3,9) (Tabela 5). 
Tabela 4

Características da assistência pré-natal e ao parto de mulheres participantes da análise de corte transversal. Município de Ribeirão Preto, São Paulo, Brasil, 2015-2016.

\begin{tabular}{|c|c|c|c|c|c|}
\hline Características do pré-natal e parto & $\begin{array}{l}\text { Total } \\
\text { n (\%) }\end{array}$ & $\begin{array}{c}\text { Mulheres com morbidade } \\
\text { materna grave * } \\
\text { n (\%) }\end{array}$ & $\begin{array}{c}\text { Mulheres sem morbidade } \\
\text { materna grave } \\
\mathbf{n}(\%)\end{array}$ & OR (IC95\%) & Valor de $p$ ** \\
\hline Gestação planejada & & & & & 0,835 \\
\hline Sim & $405(41,5)$ & $35(42,7)$ & $371(41,5)$ & $1,1(0,7-1,7)$ & \\
\hline Não & $570(58,5)$ & $47(57,3)$ & $523(58,5)$ & 1,0 & \\
\hline Realização do pré-natal & & & & & 1,000 \\
\hline Sim & $1.098(100,0)$ & $91(100,0)$ & $1.007(100,0)$ & & \\
\hline Não & $0(0,0)$ & $0(0,0)$ & $0(0,0)$ & & \\
\hline Número de consultas pré-natal & & & & & 0,450 \\
\hline$<6$ & $156(14,8)$ & $14(17,7)$ & $142(14,6)$ & $1,3(0,7-2,3)$ & \\
\hline$\geq 6$ & $897(85,2)$ & $65(82,3)$ & $832(85,4)$ & 1,0 & \\
\hline Pré-natal adequado & & & & & 0,294 \\
\hline Sim & $920(87,4)$ & $72(91,1)$ & $848(87,1)$ & 1,0 & \\
\hline Não & $122(11,6)$ & $7(8,9)$ & $126(12,9)$ & $0,7(0,3-1,5)$ & \\
\hline $\begin{array}{l}\text { Idade gestacional na 1a consulta } \\
\text { (semanas) }\end{array}$ & & & & & 0,562 \\
\hline$\leq 12$ & $537(68,3)$ & $43(71,7)$ & $494(68,0)$ & 1,0 & \\
\hline$>12$ & $249(31,7)$ & $17(28,3)$ & $232(32,0)$ & $0,8(0,5-1,5)$ & \\
\hline Local do pré-natal & & & & & 0,072 \\
\hline UBS & $901(91,2)$ & $70(85,4)$ & $831(91,7)$ & 1,0 & \\
\hline Hospital & $71(7,2)$ & $11(13,4)$ & $60(6,6)$ & $2,2(1,1-4,3)$ & \\
\hline Outros *** & $16(1,6)$ & $1(1,2)$ & $15(1,7)$ & $0,8(0,1-6,1)$ & \\
\hline Gestante de risco & & & & & $<0,001$ \\
\hline Sim & $250(25,3)$ & $48(58,5)$ & $202(22,3)$ & $4,9(3,1-7,8)$ & \\
\hline Não & $737(74,7)$ & $34(41,5)$ & $703(77,7)$ & 1 & \\
\hline Hipertensão & & & & & $<0,001$ \\
\hline Sim & $72(28,8)$ & $33(68,8)$ & $39(19,3)$ & $9,2(4,6-18,6)$ & \\
\hline Não & $178(71,2)$ & $15(31,3)$ & $163(80,7)$ & 1,0 & \\
\hline Diabetes & & & & & 0,674 \\
\hline Sim & $47(18,8)$ & $8(16,7)$ & $39(19,3)$ & $0,8(0,4-1,9)$ & \\
\hline Não & $203(81,2)$ & $40(83,3)$ & $163(80,7)$ & 1,0 & \\
\hline Sangramento na gestação & & & & & 0,631 \\
\hline Sim & $14(5,6)$ & $2(4,2)$ & $12(5,9)$ & $0,7(0,2-3,2)$ & \\
\hline Não & $236(94,4)$ & $46(95,8)$ & $190(94,1)$ & 1,0 & \\
\hline Infecção urinária & & & & & 0,866 \\
\hline Sim & $17(6,8)$ & $3(6,3)$ & $14(6,9)$ & $0,9(0,3-3,3)$ & \\
\hline Não & $234(93,2)$ & $45(93,7)$ & $188(93,1)$ & 1,0 & \\
\hline Tipo de gestação & & & & & 0,003 \\
\hline Única & $1.084(98,8)$ & $87(95,6)$ & $997(99,1)$ & 1,0 & \\
\hline Múltipla & $13(1,2)$ & $4(4,4)$ & $9(0,9)$ & $5,1(1,5-16,9)$ & \\
\hline Tipo de parto atual & & & & & $<0,001$ \\
\hline Vaginal & $742(67,6)$ & $41(45,0)$ & $701(69,6)$ & 1,0 & \\
\hline Cesariana & $356(32,4)$ & $50(55,0)$ & $306(30,4)$ & $2,8(1,8-4,3)$ & \\
\hline Trabalho de parto & & & & & $<0,001$ \\
\hline Espontâneo & $704(65,3)$ & $36(40,0)$ & $668(67,6)$ & 1,0 & \\
\hline Induzido & $213(19,8)$ & $25(27,8)$ & $188(19,0)$ & $2,5(1,4-4,2)$ & \\
\hline Ausente & $161(14,9)$ & $29(32,2)$ & $132(13,4)$ & $4,1(2,4-6,9)$ & \\
\hline
\end{tabular}

IC95\%: intervalo de 95\% de confiança; OR: odds ratio; UBS: unidades básicas de saúde.

* Condições potencialmente ameaçadoras à vida/near miss materno;

** Teste qui-quadrado ou Fisher;

*** Privado/Suplementar. 
Tabela 5

Estimativas das razões de chances (odds ratio - OR) ajustadas e correspondentes intervalos de 95\% de confiança (IC95\%) para as variáveis analisadas no modelo de regressão múltipla. Município de Ribeirão Preto, São Paulo, Brasil, $2015-2016$.

\begin{tabular}{|c|c|}
\hline Variável & OR ajustado (IC95\% *) \\
\hline \multicolumn{2}{|l|}{ Idade (anos) } \\
\hline$<35$ & 1,0 \\
\hline$\geq 35$ & $1,4(0,7-2,7)$ \\
\hline \multicolumn{2}{|l|}{ Cor da pele } \\
\hline Preta/Parda & $1,5(0,9-2,5)$ \\
\hline Outras ** & 1,0 \\
\hline \multicolumn{2}{|l|}{ Trabalho remunerado } \\
\hline Sim & $0,8(0,5-1,2)$ \\
\hline Não & 1,0 \\
\hline \multicolumn{2}{|l|}{ Total de partos anteriores } \\
\hline 0 & 1,0 \\
\hline $1-2$ & $0,7(0,4-1,3)$ \\
\hline$\geq 3$ & $1(0,5-2,1)$ \\
\hline \multicolumn{2}{|l|}{ Local do pré-natal } \\
\hline UBS & 1,0 \\
\hline Hospital de referência & $0,6(0,3-1,5)$ \\
\hline Outro *** & $0,5(0,1-4,5)$ \\
\hline \multicolumn{2}{|l|}{ Gestante de risco } \\
\hline Sim & $4,5(2,7-7,7)$ \\
\hline Não & 1,0 \\
\hline \multicolumn{2}{|l|}{ Tipo de gestação } \\
\hline Única & 1,0 \\
\hline Múltipla & $2,3(0,5-9,5)$ \\
\hline \multicolumn{2}{|l|}{ Tipo de parto } \\
\hline Vaginal & 1,0 \\
\hline Cesariana & $1,9(1-3,6)$ \\
\hline \multicolumn{2}{|l|}{ Trabalho de parto } \\
\hline Espontâneo & 1,0 \\
\hline Induzido & $2,1(1,2-3,9)$ \\
\hline Ausente & $1,7(0,8-3,7)$ \\
\hline
\end{tabular}

UBS: unidades básicas de saúde.

* Obtido por regressão logística múltipla;

** Branca, indígena e oriental/amarela;

*** Privado/Suplementar.

\section{Discussão}

A razão de near miss materno observada (3,6 casos por mil nascidos vivos) foi inferior aos indicadores encontrados em pesquisas realizadas no Irã, Oriente Médio, Ruanda, Paquistão e em outros estudos desenvolvidos em países de renda média 9,18,19,20,21.

Deve-se destacar que Ribeirão Preto possui alto IDH (0,8), caracterizando-o como um município com elevado desenvolvimento social e econômico, além de possuir indicadores educacionais acima da média nacional 14 .

Além disso, a cidade é conhecida por possuir uma rede de serviços de saúde de qualidade, desde a atenção primária até o nível terciário, sendo uma das maternidades pesquisadas a referência para toda a região, inclusive para o Estado de São Paulo, em atendimento à gestação de alto risco.

As melhores condições socioeconômicas e a existência de uma rede de serviços que atenda às demandas das mulheres são aspectos diretamente relacionados à melhoria da saúde materna 22, o que 
pode ter relação direta com a menor ocorrência de near miss materno por grupo de nascidos vivos observada nesta análise.

Estudo de tendência temporal realizado com dados das diferentes regiões brasileiras entre 2000 e 2014 mostrou aumento da razão do near miss materno nas regiões Norte e Nordeste e decréscimo do indicador nas regiões Sul e Sudeste, áreas que, no geral, são caracterizadas por melhor assistência obstétrica, além de maior desenvolvimento socioeconômico 23.

As complicações hipertensivas foram as mais frequentes nas maternidades pesquisadas, e essa também é a principal causa de adoecimento e óbito materno no Brasil e em diversos países da África, Ásia, América Latina e Oriente Médio 10,24,25.

As patologias hipertensivas, quando identificadas precocemente e manejadas adequadamente durante o pré-natal, diminuem a probabilidade de desencadear graves complicações durante o ciclo gravídico-puerperal. Com isso, elevadas taxas de hipertensão grave e pré-eclâmpsia podem evidenciar a necessidade de melhoria do rastreio das complicações hipertensivas durante o pré-natal 18.

Todas as mulheres pesquisadas fizeram o acompanhamento pré-natal, e aproximadamente $90 \%$ realizaram seis ou mais consultas, mas a alta cobertura não foi suficiente para evitar a elevada frequência dos distúrbios hipertensivos graves no ciclo gravídico-puerperal. Por isso, a avaliação da assistência obstétrica deve ir além das análises de cobertura, sendo a qualidade do cuidado ofertado para as gestantes uma discussão fundamental e necessária para a melhoria da saúde materna 26 .

Observou-se associação da morbidade materna grave com o fato de a mulher ser classificada como grupo de risco, relação esperada, mas que mostra a necessidade de análise do risco obstétrico a cada consulta de pré-natal, não de uma classificação estanque a ser realizada apenas no primeiro momento da gestação 25 .

Neste contexto, a estruturação de um projeto terapêutico individualizado, principalmente para as gestantes de risco, além da garantia de serviços de referência de qualidade e em tempo oportuno em caso de agravamento, são essenciais para a prevenção das morbidade materna grave e do óbito materno.

Observou-se associação da morbidade materna grave com o trabalho de parto induzido e gestação de risco, sendo tais associações encontradas em estudos realizados no Brasil e na Austrália $5,27,28$. Todavia, esta análise trata-se de um estudo transversal; neste contexto, as duas variáveis foram coletadas no mesmo momento, não sendo possível falar que a indução contribui para a ocorrência das complicações maternas ou que a indução foi um manejo adotado em decorrência das morbidades.

A indução do trabalho de parto, quando realizada adequadamente, é um procedimento de proteção da saúde materna em situações de risco 29 , por isso, a associação entre complicações graves e induzir o parto pode inclusive indicar adequada qualidade da assistência obstétrica.

Não foram encontradas associações entre morbidade materna grave e variáveis sociodemográficas, o que acontece principalmente em regiões mais carentes. A não existência dessa relação é um perfil observado nos países que estão na fase III da transição obstétrica (RMM entre 299 e 50 óbitos por 100 mil nascidos vivos), que é o caso do Brasil 30.

Os óbitos maternos que ocorrem nesse grupo de países estão relacionados principalmente à qualidade da assistência obstétrica e não às precárias condições de vida 31 , o que reforça a importância em se melhorar o cuidado ofertado no ciclo gravídico-puerperal às mulheres brasileiras.

Uma vantagem deste estudo é a possibilidade de analisar a relação entre morbidade materna grave e aspectos distintos que vão desde os sociodemográficos até os assistenciais, viabilizando compreender as especificidades que podem influenciar ou não no adoecimento materno.

Entretanto, é importante que estudos como este sejam realizados nas diferentes localidades brasileiras, pois os achados do município pesquisado podem ser distintos daqueles a serem encontrados em locais mais carentes, perfil de grande parte dos municípios brasileiros, principalmente aqueles localizados nas regiões Norte e Nordeste.

$\mathrm{O}$ entendimento dos fatores associados à morbidade materna grave em regiões distintas pode contribuir para a compreensão da saúde materna de cada localidade, conhecimento fundamental para a estruturação de uma rede assistencial e de serviços de saúde preparados para o rastreamento e tratamento precoce das complicações maternas, o que pode impactar diretamente a redução dos indicadores de morbimortalidade materna. 
Uma limitação está relacionada ao fato de não terem sido estudadas mulheres com menos de 18 anos, pois o PRENACEL pesquisou apenas aquelas com 18 ou mais e as adolescentes formam um grupo que sabidamente pode apresentar um maior risco obstétrico.

Outra limitação da pesquisa foi o fato de não terem entrado na análise transversal os casos de aborto e gestação ectópica, pois foram pesquisadas apenas as mulheres que integravam o estudo PRENACEL, que tinha como um dos critérios de inclusão mulheres que tiveram parto.

Também foram retiradas as mulheres que integravam o grupo intervenção da pesquisa PRENACEL após a verificação da possibilidade de a intervenção apresentar efeito protetor, reduzindo a ocorrência de morbidade materna grave, com isso, a permanência dessas mulheres na análise transversal poderia trazer viés aos resultados do estudo.

Para checar se a retirada das mulheres do grupo intervenção poderia comprometer a presente análise, verificou-se a existência de diferenças estatisticamente significantes ao comparar as variáveis pesquisadas entre as mulheres intervenção e não intervenção, não havendo nenhuma diferença significante, o que reduz a possibilidade de a retirada dessas mulheres enviesar a análise apresentada neste artigo.

\section{Conclusões}

Os resultados da pesquisa mostram que a frequência dos casos de near miss Materno entre as mulheres pesquisadas foi menor se comparado às pesquisas realizadas em diferentes outros países, o que pode ocorrer devido aos elevados indicadores socioeconômicos e da boa organização da rede de serviços de saúde existente no município.

Os distúrbios hipertensivos destacam-se entre as causas de morbidade materna grave, o que evidencia a necessidade de melhorias no rastreio e tratamento dos elevados níveis pressóricos das gestantes nos serviços de pré-natal, bem como adequada assistência obstétrica para os casos mais graves.

A associação entre morbidade materna grave e gestação de risco sinaliza que a análise da existência de riscos deve ocorrer durante todo o período gravídico, pois não se sabe em qual momento da gestação a complicação pode acontecer, e esse acompanhamento contínuo pode viabilizar a prevenção e o tratamento precoce do adoecimento grave e do óbito materno.

A observação da associação entre indução do trabalho de parto e morbidade materna grave pode indicar uma adequada qualidade da assistência, já que a interrupção da gestação em tempo oportuno pode ser essencial para a preservação da saúde materna e/ou fetal.

Entretanto, para a afirmação dessa relação causal, bem como para a análise da associação entre morbidade materna grave e demais variáveis, é necessária a realização de estudos que permitam a coleta de informações em diferentes momentos da linha temporal, viabilizando o levantamento das variáveis que de fato contribuam para a ocorrência de complicações maternas graves. 


\section{Colaboradores}

M. S. Andrade, J. P. Souza e E. M. Vieira contribuíram na concepção do projeto, análise e interpretação dos dados, redação do artigo, revisão crítica relevante do conteúdo intelectual e aprovação da versão final. L. P. Bonifácio, J. A. C. Sanchez, F. S. Zaratini, A. C. A. Franzon, V. N. Pileggi, G. C. Braga e $\mathrm{M}$. Fernandes contribuíram na redação do artigo e revisão crítica relevante do conteúdo intelectual. L. Oliveira-Ciabati contribuiu na análise e interpretação dos dados, redação do artigo. C. S. Vieira contribuiu na concepção do projeto, análise e interpretação dos dados, redação do artigo e revisão crítica relevante do conteúdo intelectual.

\section{Informações adicionais}

ORCID: Magna Santos Andrade (0000-00020557-2169); Livia Pimenta Bonifacio (0000-00024309-0304); Jazmin Andrea Cifuentes Sanchez (0000-0002-4552-0443); Livia Oliveira-Ciabati (0000-0002-7163-9456); Fabiani Spessoto Zaratini (0000-0002-7758-4388); Ana Carolina Arruda Franzon (0000-0003-4333-3326); Vicky Nogueira Pileggi (0000-0003-2284-010X); Giordana Campos Braga (0000-0002-0719-056X); Mariana Fernandes (0000-0002-4551-7017); Carolina Sales Vieira (0000-0003-2933-5755); João Paulo Souza (00000002-2288-4244); Elisabeth Meloni Vieira (00000002-5229-3904).

\section{Agradecimentos}

Ao Programa Ciência sem Fronteiras (CSF-PAJT 2514/2013) da Coordenação de Aperfeiçoamento de Pessoal de Nível Superior/Conselho Nacional de Desenvolvimento Científico e Tecnológico (no processo no 23038.007622/2013-60) e Fundação de Amparo à Pesquisa do Estado de São Paulo (processo 2015/16932-5) pelo financiamento, e à Organização Mundial da Saúde pelo apoio institucional.

\section{Referências}

1. World Health Organization. Trends in maternal mortality 2000 to 2017: estimates by WHO, UNICEF, UNFPA, World Bank Group and the United Nations Population https://apps.who. int/iris/handle/10665/327596 (acessado em Nov/2019).

2. United Nations. Maternal mortality and morbidity and human rights. https://www.ohchr. org/Documents/Issues/Women/WRGS/ OnePagers/Maternal_mortality_morbidity. pdf (acessado em Ago/2018).

3. Departamento de Informática do SUS. Indicadores de mortalidade e indicadores de nascidos vivos. http://tabnet.datasus.gov.br/cgi/ tabcgi.exe?sinasc/cnv/nvuf.def (acessado em Jun/2021).

4. Departamento de Análise de Situação de Saúde, Secretaria de Vigilância em Saúde, Ministério da Saúde. Saúde Brasil 2011: uma análise da situação de saúde e a vigilância da saúde da mulher. Brasília: Ministério da Saúde; 2012.

5. Dias MAB, Domingues RMSM, Schilithz AOC, Nakamura-Pereira M, Diniz CSG, Brum IR, et al. Incidência do near miss materno no parto e pós-parto hospitalar: dados da pesquisa Nascer no Brasil. Cad Saúde Pública 2014; 30 Suppl 1: S169-81.

6. Haddad SM, Cecatti JC, Souza JP, Sousa MH, Parpinelli MA, Costa ML, et al. Applying the maternal near miss approach for the evaluation of quality of obstetric care: a worked example from a Multicenter Surveillance Study. Biomed Res Int 2014; 2014:989815.

7. Say L, Souza JP, Pattinson RC. Maternal near miss - towards a standard tool for monitoring quality of maternal health care. Best Pract Res Clin Obstet Gynaecol 2009; 23:287-96.

8. Pattinson R, Say L, Souza JP, Broek N, Rooney C. WHO maternal death and near-miss classifications. Bull World Health Organ 2009; 87:734

9. Souza JP, Gülmezoglu AM, Vogel J, Carroli G, Lumbiganon P, Qureshi Z, et al. Moving beyond essential interventions for reduction of maternal mortality (the WHO Multicountry Survey on Maternal and Newborn Health): a crosssectional study. Lancet 2013; 381:1747-55.

10. Cecatti JG, Costa ML, Haddad SM, Parpinelli MA, Souza JP, Sousa MH, et al. Network for surveillance of severe maternal morbidity: a powerful national collaboration generating data on maternal health outcomes and care. BJOG 2016;123:946-53.

11. Domingues RMSM, Dias MAB, Schilithz AOC, Leal MC. Factors associated with maternal near miss in childbirth and the postpartum period: findings from the birth in Brazil National Survey, 2011-2012. Reprod Health 2016; 13 Suppl 3:187-97.

12. Firoz T, Chou D, Dadelszen P, Agrawal P, Vanderkruik R, Tunçalp O, et al. Measuring maternal health: focus on maternal morbidity. Bull World Health Organ 2013; 91:794-96. 
13. Oliveira-Ciabati L, Vieira CS, Franzon ACA, Alves D, Zaratini FS, Braga GC, et al. PRENACEL - a mHealth messaging system to complement antenatal care: a cluster randomized trial Reprod Health 2017; 14:146.

14. Instituto Brasileiro de Geografia e Estatística. População e economia. Brasil/São Paulo/ Ribeirão Preto. 2020. https://cidades.ibge.gov. $\mathrm{br} / \mathrm{brasil} / \mathrm{sp} /$ ribeirao-preto/panorama (acessado em Nov/2020).

15. Associação Brasileira de Empresas de Pesquisa. Critério de classificação econômica Brasil - 2014. http://www.abep.org/criterio-brasil (acessado em Jun/2015)

16. Gomes MNA, Santos LKO, Matos MAB, Lopes Prr, Chomatas ERV, Barra RP, et al. Nota técnica para organização da rede de atenção à saúde com foco na atenção primária á saúde e na atenção ambulatorial especializada. Saúde da mulher na gestação, parto e puerpério. São Paulo: Ministério da Saúde/Sociedade Beneficente Israelita Brasileira Albert Einstein; 2019.

17. Harris PA, Taylor R, Thielke R, Payne J, Gonzalez N, Conde JG. Research electronic data capture (REDCap): a metadata-driven methodology and workflow process for providing translational research informatics support. J Biomed Inform 2009; 42:377-81.

18. Ghazivakili Z, Lotfi R, Kabir K, Norouzi Nia R, Rajabi Naeeni M. Maternal near miss approach to evaluate quality of care in Alborz province, Iran. Midwifery 2016; 41:118-24.

19. Bashour H, Saad-Haddad G, DeJong J, Ramadan MC, Hassan S, Breebaart M, et al. A cross sectional study of maternal 'near-miss' cases in major public hospitals in Egypt, Lebanon, Palestine and Syria. BMC Pregnancy Childbirth 2015; 15:296.

20. Rulisa S, Umuziranenge I, Small M, van Roosmalen J. Maternal near miss and mortality in a tertiary care hospital in Rwanda. BMC Pregnancy Childbirth 2015; 15:203.

21. Mazhar SB, Batool A, Emanuel A, Khan AT, Bhutta S. Severe maternal outcomes and their predictors among Pakistani women in the WHO Multicountry Survey on Maternal and Newborn Health. Int J Gynaecol Obstet 2015; 129:30-3.

22. Pacagnella RC, Cecatti JG, Parpinelli MA, Sousa MH, Haddad SM, Costa ML, et al. Delays in receiving obstetric care and poor maternal outcomes: results from a national multicentre cross-sectional study. BMC Pregnancy Childbirth 2014; 14:159.
23. Carvalho BAS, Andrade AGBF, Dantas AS, Figueiredo IM, Silva JA, Rosendo TS, et al. Tendência temporal do Near Miss materno no Brasil entre 2000 e 2012. Rev Bras Saúde Mater Infant (Online) 2019; 19:115-24.

24. Silva JMP, Fonseca SC, Dias MAB, Izzo AS, Teixeira GP, Belfort PP. Conceitos, prevalência e características da morbidade materna grave, near miss, no Brasil: revisão sistemática. Rev Bras Saúde Mater Infant (Online) 2018; 18:7-35.

25. Soares FM, Pacagnella RC, Tunçalp O, Cecatti JG, Vogel JP, Togoobaatar G, et al. Provision of intensive care to severely ill pregnant women is associated with reduced mortality: results from the WHO Multicountry Survey on Maternal and Newborn Health. Int J Gynaecol Obstet 2020; 150:346-53.

26. Andrade MS, Vieira EM. Itinerários terapêuticos de mulheres com morbidade materna grave. Cad Saúde Pública 2018; 34:e00091917.

27. Pacheco AJ, Katz L, Souza AS, de Amorim MM. Factors associated with severe maternal morbidity and near miss in the São Francisco Valley, Brazil: a retrospective, cohort study. BMC Pregnancy Childbirth 2014; 14:91.

28. Knigth M, Acosta C, Brocklehurst P, Cheshire A, Fitzpatrick K, Hinton L, et al. Beyond maternal death: improving the quality of maternal care through national studies of 'near miss' maternal morbidity. Southampton: NIHR Journals Library; 2016

29. Departamento de Ações Programáticas Estratégicas, Secretaria de Atenção à Saúde, Ministério da Saúde. Gestação de alto risco: manual técnico. Brasília: Ministério da Saúde; 2012. (Série A. Normas e Manuais Técnicos).

30. Chaves SC, Cecatti JG, Carroli G, Lumbiganon $\mathrm{P}$, Hogue CJ, Mori R, et al. Obstetric transition in the World Health Organization Multicountry Survey on Maternal and Newborn Health: exploring pathways for maternal mortality reduction. Rev Panam Salud Pública 2015; 37:203-10. 


\section{Abstract}

This study investigated the factors associated with serious maternal morbidity (SMM) in women seen at public maternity hospitals in Ribeirão Preto, São Paulo State, Brazil. This was a cross-section analytical quantitative study. Participation included 1,098 postpartum women who had given birth at one of the four maternity hospitals in the municipality. Data were collected from August 3, 2015, to February 2, 2016, using face-to-face interviews and data obtained from patient records and prenatal cards. The dependent variable for data analysis was the serious maternal morbidity, that is, when the woman was classified as maternal near miss or potentially life-threatening condition based on WHO eligibility criteria. The study calculated the maternal near miss ratio, odds ratio (OR), 95\% confidence interval (95\%CI), and multiple logistic regression. The maternal near miss ratio was 3.6 cases per 1,000 live births. Complications occurred mainly during pregnancy (53.8\%), and hypertensive disorders were the most frequent (49.4\%). Multiple regression analysis showed an association between serious maternal morbidity and high-risk pregnancy $(O R=4.5$, 95\% CI: 2.7-7.7) and induced labor $(O R=2.1$, 95\%CI: 1.2-3.9). The occurrence of serious maternal morbidity mainly during pregnancy, featuring hypertensive syndromes, points to the need for better screening and management of high blood pressure in the prenatal period. The association between serious maternal morbidity and high-risk pregnancy also calls attention to prenatal care, for the demand for greater care for women classified as having gestational risk. Quality of care is a key point for dealing with maternal morbidity and mortality in Brazil.

Healthcare Near Miss; Maternal Death; Morbidity; Maternal Health

\section{Resumen}

Este estudio investigó los factores asociados a la morbilidad materna grave entre mujeres atendidas en maternidades públicas del municipio de Ribeirão Preto, São Paulo, Brasil. Se trata de un estudio cuantitativo, analítico, transversal. Participaron 1.098 puérperas con parto en una de las cuatro maternidades públicas del municipio. La recogida de datos se produjo entre el 3 de agosto de 2015 al 2 de febrero de 2016, mediante entrevistas cara a cara, obtención de información de los historiales y cartillas de las gestantes. Para el análisis de datos, se consideró como variable dependiente la ocurrencia de morbilidad materna grave, o sea, cuando la mujer era clasificada como near miss materno o condición potencialmente amenazadora para la vida, a partir de los criterios de elegibilidad de la Organización Mundial de Salud. Se calculó la razón del near miss materno, odds ratio (OR), intervalo de 95\% de confianza (IC95\%) y regresión logística múltiple. La razón de near miss materno fue de 3,6 casos por 1.000 nacidos vivos. Las complicaciones se produjeron principalmente en el embarazo $(53,8 \%)$ y las alteraciones hipertensivas fueron las más frecuentes (49,4\%). El análisis de regresión múltiple mostró asociación entre morbilidad materna grave y gestación de riesgo $(O R=$ 4,5; IC95\%: 2,7-7,7) y con trabajo de parto inducido (OR = 2, 1; IC95\%: 1,2-3,9). La ocurrencia de morbilidad materna grave, principalmente en la gestación, resaltando los sindromes hipertensivos, apunta la necesidad de un mejor rastreo y gestión de la elevación de los niveles presión arterial en el periodo prenatal. La asociación entre morbilidad materna grave y gestación de riesgo también se remonta al período prenatal, con el fin de que se preste una mayor atención a las mujeres clasificadas como de riesgo gestacional. La calidad de la asistencia es un punto clave para combatir la morbimortalidad materna en el país.

Near Miss Salud; Muerte Materna; Morbilidad; Salud Materna
Recebido em 28/Jan/2021

Versão final reapresentada em 07/Jul/2021

Aprovado em 16/Jul/2021 